\title{
SASAKIAN GEOMETRY AND EINSTEIN METRICS ON SPHERES
}

\author{
CHARLES P. BOYER AND KRZYSZTOF GALICKI
}

\section{INTRODUCTION}

This paper is based on a talk presented by the first author at the Short Program on Riemannian Geometry that took place at the Centre de Recherche Mathématiques, Université de Montréal, during the period June 28-July 16, 2004. It is a report on our joint work with János Kollár BGK03 concerning the existence of an abundance of Einstein metrics on odd dimensional spheres, including exotic spheres. The article appeared electronically in September of 2003, and answered in the affirmative the heretofore open question of the existence of Einstein metrics on exotic spheres. Evan Thomas helped us with the computer programs BGKT03.

1.1. A Brief History of our Approach. For more than ten years the authors and their collaborators have established a program employing Sasakian geometry to prove the existence of Einstein metrics on compact odd dimensional manifolds. It began BGM93b, BGM93a, BGM94 with the study of 3-Sasakian manifolds which are automatically Einstein. The main technique used to establish the existence of Einstein 3-Sasakian metrics was that of symmetry reduction, a method well-known in symplectic geometry. This work reached its pinnacle in the Inventiones paper BGMR98, where we constructed all toric 3-Sasakian manifolds in dimension 7. In particular, we gave the first examples of Einstein metrics on compact simply-connected manifolds with arbitrary second Betti number. A survey of the results in this area appeared later in BG99.

In BG00 we began the study of Sasakian-Einstein structures. In this more general case the symmetry reduction yielded very few results, and another method was needed. In late 1999 we noticed the preprint version of the paper [DK01 by Demailly and Kollár on the LANL ArXivs which gave sufficient algebraic conditions for the vanishing of the obstructions to solving the Monge-Ampère equations for Kähler orbifolds with positive first Chern class. More importantly, Demailly and Kollár exhibited explicit examples of log del Pezzo surfaces with orbifold singularities as certain hypersurfaces in weighted projective spaces. This entailed showing that the singularities of the pair (log del Pezzo surface, anticanonical divisor) is relatively mild, a condition known in the algebraic geometry literature as Kawamata log terminal, or klt for short. Their article prompted us to develop a method BG01] which uses the links of hypersurface singularities of weighted homogeneous polynomials to give the first non-regular examples of Sasakian-Einstein metrics in dimension five. Shortly thereafter we received a preliminary version of [JK01] in which many more explicit examples of log del Pezzo surfaces with a Kähler-Einstein orbifold metric were given. We teamed up with our algebraic geometer colleague Michael Nakamaye [BGN03a, BGN02, BG03b] to apply the method of BG01 to prove the existence of many Sasakian-Einstein metrics on the connected sums $\# k\left(S^{2} \times S^{3}\right)$ for $1 \leqslant k \leqslant 9$. (And very recently Kollár Kol04b has proven that Sasakian-Einstein metrics exist on $k \#\left(S^{2} \times S^{3}\right)$ for all $k \geqslant 6$. In fact, he has shown 
that there are infinitely many $2(k-1)$-dimensional families of Sasakian-Einstein metrics on $k \#\left(S^{2} \times S^{3}\right)$. Thus, all connected sums of $S^{2} \times S^{3}$ admit many Sasakian-Einstein metrics.) However, the question of how to handle the klt conditions when the Kähler orbifolds have branch divisors alluded us. This occurs, for example, when the links are homotopy spheres, and we had early on recognized that it was a potential approach to proving the existence of an Einstein metric on an exotic sphere. In fact, we already had what we thought was a good candidate for a Sasakian-Einstein structure on an exotic sphere. Then in the summer of 2003 we began serious discussions with János Kollár who understood the singularities with branch divisors present. We not only were able to verify that our original candidate provided the first example of an Einstein metric on an exotic sphere, but we found, much to our surprise and delight, that Einstein metrics exist in huge numbers on many homotopy spheres. We would like to take this opportunity to thank János for taking an early interest in our work, and then providing much of the essential ingredients of our joint papers. We also thank him for useful comments concerning this expository paper.

1.2. A History of Einstein Metrics on Spheres. The name comes from Einstein famous work creating general relatively. Working with the indefinite Lorenzian signature of 4dimensional space-time, Einstein's reasoning went roughly as follows: the total amount of energy and momentum in the universe should equal the curvature of the universe. Energy and momentum is represented by a symmetric 2-tensor $T_{\mu \nu}$, and there are precisely two natural symmetric 2-tensors in Riemannian (Lorenzian) geometry, the Ricci curvature, $R_{\mu \nu}$, and the Riemannian metric itself $g_{\mu \nu}$. Hence, in tensor indices one has the equation

$$
G_{\mu \nu}:=R_{\mu \nu}-\frac{1}{2} s g_{\mu \nu}=\kappa T_{\mu \nu}-\Lambda g_{\mu \nu},
$$

where $G_{\mu \nu}$ is sometimes called the Einstein tensor, $s=\sum_{\mu, \nu} g^{\mu \nu} R_{\mu \nu}$ is the scalar curvature, and $\kappa, \Lambda$ are constants. If one assumes that the only energy in the universe is gravitational, then $T_{\mu \nu}=0$. Actually, Einstein originally had the "cosmological constant" $\Lambda=0$ as well, but he then inserted a non-zero value of $\Lambda$ to obtain a solution representing a static universe. Later when Hubble discovered that the universe was actually expanding, he called inserting $\Lambda$ "the biggest blunder of my life". Ironically, the recently discovered acceleration in the expansion of the universe again suggests a non-zero value of $\Lambda$ in order to account for the so-called "dark energy" which represents a type of anti-gravitational pressure that causes the acceleration in the expansion of the universe. So in the absence of other forces, the Einstein equations become $R_{\mu \nu}=\left(\frac{1}{2} s-\Lambda\right) g_{\mu \nu}$. This is the origin of the mathematicians' well known definition of an Einstein metric, viz.

Definition 1.1: A Riemannian metric $g$ is said to be an Einstein metric if $\mathrm{Ric}_{g}=\lambda g$ for some constant $\lambda$.

The fact that $\lambda$ is a constant is a consequence of the Bianchi identities. The best known examples of Einstein metrics are metrics of constant curvature, and long before Einstein gave us his modern view of the universe, even before Riemann's "epoch-making" essay on the foundation of geometry, Gauss had studied spherical geometry and understood the "round sphere metric" at least in dimension 2. More than one hundred years had passed since the deaths of both Gauss and Riemann before an example of an Einstein metric other than the round sphere metric was shown to exist on a sphere. In 1973, Gary Jensen Jen73 proved the existence of what later became known as a "squashed" metric on $S^{4 n+3}$. The next 25 
years brought about very little change with just a handful of new results in the subject. We very briefly summarize this history:

- The standard metric on $S^{n}$ (Gauss-Riemann).

- The squashed metrics on $S^{4 n+3}$ (Jensen, 1973 [Jen73]).

- A homogeneous Einstein metric on $S^{15}$ (Bourguignon and Karcher, 1978 [BK78]).

- These are all homogeneous Einstein metrics on $S^{n}$ and they are the only such metrics up to homothety (Ziller, 1982 [Zil82]).

- Infinite sequences of inhomogeneous Einstein metrics on $S^{5}, S^{6}, S^{7}, S^{8}$ and $S^{9}$ (Böhm, 1998 Böh98]).

In contrast, we have been able to prove the following striking results:

Theorem 1.2: BGK03 There exists inequivalent families of Einstein metrics on all odd dimensional spheres, the number of deformation classes of Sasakian-Einstein structures grows double exponentially with dimension. Some of these have moduli, the largest of which the number of moduli grows double exponentially with dimension. There exist Einstein metrics on all homotopy spheres in dimension 7 and all homotopy spheres in dimension $4 n+1$ that bound parallelizable manifolds. We obtain at least 68 inequivalent deformation classes of Sasakian-Einstein metrics on $S^{5}$, and 8610 deformation classes of Sasakian-Einstein metrics on homotopy spheres that are homeomorphic to $S^{7}$.

All the Einstein metrics in this theorem are actually Sasakian-Einstein .

Theorem 1.3: BGKT03] There exists Einstein metrics on all homotopy spheres in dimension 11 and 15 that bound parallelizable manifolds, that is, all 992 homotopy spheres in $b P_{12}$ and all 8128 homotopy spheres in $b P_{16}$ admit Sasakian-Einstein metrics. The distribution of the 8610 deformation classes of Sasakian-Einstein metrics on homotopy spheres $\Sigma_{i}$ in dimension 7 is given by $\left(n_{1}, \ldots, n_{28}\right)=(376,336,260,294,231,284,322,402,317,309,252$, 304, 258, 390, 409, 352, 226, 260, 243, 309, 292, 452, 307, 298, 230, 307, 264, 353), where $n_{i}$ is the number of deformation classes of Sasakian-Einstein metrics on $\Sigma_{i}$, and $\Sigma_{1}$ is the Milnor generator. For each diffeomorphism type in dimension 7 there exist Sasakian-Einstein metrics with moduli. The standard $S^{7}=\Sigma_{28}$ admits an 82-dimensional family of Sasakian-Einstein metrics.

As mentioned above Böhm Böh98 found infinite sequences of Einstein metrics on certain spheres, but it appears that our work BGK03, BGKT03 gives the first examples of Einstein metrics on spheres with a positive lower bound on the dimension of the moduli space. See Wan99 for a very nice review for obtaining Einstein metrics from symmetry techniques or bundle constructions.

\section{Homotopy Spheres}

In 1956 John Milnor Mil56 stunned the mathematical world by constructing smooth manifolds $\Sigma^{7}$ that are homeomorphic but not diffeomorphic to $S^{7}$. This began the field of Differential Topology. For homotopy spheres the situation was described in a foundational paper by Kervaire and Milnor in 1963 KM63. We briefly summarize their results. Kervaire and Milnor defined an Abelian group $\Theta_{n}$ which consists of equivalence classes of homotopy spheres of dimension $n$ that are equivalent under oriented h-cobordism. By Smale's h-cobordism theorem this implies equivalence under oriented diffeomorphism. The group operation on $\Theta_{n}$ is connected sum. Now $\Theta_{n}$ has an important subgroup $b P_{n+1}$ which consists 
of equivalence classes of those homotopy spheres which are the boundary of a parallelizable manifold. It is the subgroup $b P_{2 n}$ that interests us. Kervaire and Milnor proved:

- $b P_{2 m+1}=0$.

- $b P_{4 m}(m \geqslant 2)$ is cyclic of order $\left|b P_{4 m}\right|=2^{2 m-2}\left(2^{2 m-1}-1\right)$ numerator $\left(\frac{4 B_{m}}{m}\right)$, where $B_{m}$ is the $m$-th Bernoulli number. Thus, for example $\left|b P_{8}\right|=28,\left|b P_{12}\right|=$ $992,\left|b P_{16}\right|=8128,\left|b P_{20}\right|=130,816$.

- $b P_{4 m+2}$ is either 0 or $\mathbb{Z}_{2}$.

Determining which $b P_{4 m+2}$ is $\{0\}$ and which is $\mathbb{Z}_{2}$ has proven to be difficult in general, and is still not completely understood. If $m \neq 2^{i}-1$ for any $i \geqslant 3$, then Browder Bro69. proved that $b P_{4 m+2}=\mathbb{Z}_{2}$. However, $b P_{4 m+2}$ is the identity for $m=1,3,7,15$, due to several people [MT67, BJM84]. See Lan00] for a recent survey of results in this area and complete references. The answer is still unknown in the remaining cases. Using surgery Kervaire was the first to show that there is an exotic sphere in dimension 9. His construction works in all dimensions of the form $4 m+1$, but as just discussed they are not always exotic.

\section{Sasakian GeOmetry}

What are now called Sasakian structures were first introduced by Sasaki in 1960 Sas60 and were subsequently developed mainly in the Japanese literature (See [YK84 and Bla76, Bla02 for complete references). They turn out to be an odd dimensional version of Kähler structures.

Proposition/Definition 1: Then a Riemannian manifold $(M, g)$ is called a Sasakian manifold if any one, hence all, of the following equivalent conditions hold:

(i) There exists a Killing vector field $\xi$ of unit length on $M$ so that the tensor field $\Phi$ of type $(1,1)$, defined by $\Phi(X)=-\nabla_{X} \xi$, satisfies the condition

$$
\left(\nabla_{X} \Phi\right)(Y)=g(X, Y) \xi-g(\xi, Y) X
$$

for any pair of vector fields $X$ and $Y$ on $M$.

(ii) There exists a Killing vector field $\xi$ of unit length on $M$ so that the Riemann curvature satisfies the condition

$$
R(X, \xi) Y=g(\xi, Y) X-g(X, Y) \xi
$$

for any pair of vector fields $X$ and $Y$ on $M$.

(iii) The metric cone on $M(C(M), \bar{g})=\left(\mathbb{R}_{+} \times M, d r^{2}+r^{2} g\right)$ is Kähler.

We define a 1 -form $\eta$ by $\eta(X)=g(\xi, X)$. Then it follows that $\eta$ is a contact 1-form (i.e., $\eta \wedge(d \eta)^{n} \neq 0$ ) and $\xi$ is its Reeb vector field. The tensor field $\Phi$ restricted to the contact subbundle $\mathcal{D}=$ ker $\eta$ is an integrable almost complex structure defining a strictly pseudoconvex CR structure. Thus, a Sasakian structure $\mathcal{S}$ is described by tensor fields $(\xi, \eta, \Phi, g)$, which describe a 1-dimensional Riemannian foliation $\mathcal{F}_{\xi}$, called the characteristic foliation, whose transverse geometry is Kähler.

Given a Sasakian structure $\mathcal{S}=(\xi, \eta, \Phi, g)$ on $M$ there are many Sasakian structures that can be obtained in several different ways. First adding a basic (with respect to $\mathcal{F}_{\xi}$ ) 1 -form $\zeta$ to $\eta$ gives a new Sasakian structure. Second when there are non-trivial symmetries one can deform the foliation to obtain new Sasakian structures. Although a homothety of a Sasakian metric is no longer Sasakian, there is a "transverse homothety" YK84, BG05] 
given by $(\xi, \eta, \Phi, g) \mapsto\left(a^{-1} \xi, a \eta, \Phi, a g+\left(a^{2}-a\right) \eta \otimes \eta\right)$ whose image is a Sasakian structure. There is also a conjugate Sasakian structure defined by $\mathcal{S}^{c}=(-\xi,-\eta,-\Phi, g)$.

We are particularly interested in

Definition 3.1: A Sasakian manifold $(M, g)$ is Sasakian-Einstein if the metric $g$ is also Einstein.

Any Sasakian-Einstein metric must have positive scalar curvature, which follows from $\operatorname{Ric}(X, \xi)=2 n \eta(X)$. So any complete Sasakian-Einstein manifold must be compact with finite fundamental group. The following is essentially due to Tanno [Tan70]

Lemma 3.2: Let $\mathcal{S}=(\xi, \eta, \Phi, g)$ and $\mathcal{S}^{\prime}=\left(\xi^{\prime}, \eta^{\prime}, \Phi^{\prime}, g\right)$ be two Sasakian structures sharing the same Riemannian metric $g$, and suppose that $(M, g)$ is not a space form with sectional curvature equal to 1 . Then either

(i) $\mathcal{S}^{\prime}=\mathcal{S}$,

(ii) $\mathcal{S}^{\prime}=\mathcal{S}^{c}$ the conjugate Sasakian structure, or

(iii) $\mathcal{S}$ and $\mathcal{S}^{\prime}$ are part of a 3-Sasakian structure.

We refer to [BG99] for the definition of a 3-Sasakian structure. The upshot is that the types of Sasakian structures studied here are not compatible with a 3-Sasakian structure. Lemma 3.2 is important for the inequivalence of the Einstein metrics we obtain. See Lemma 5.2 below.

3.1. Relation with Algebraic Geometry. The following two theorems generalize results of Boothby-Wang BW58, Hatakeyama Hat63, and Kobayashi Kob63 to the case where the quotient is an orbifold. A very brief discussion of orbifolds will then follow.

Theorem 3.3: BG00] Let $(M, g)$ be a compact quasi-regular Sasakian manifold of dimension $2 n+1$, and let $Z$ denote the space of leaves of the characteristic foliation $\mathcal{F}_{\xi}$. Then

(i) The leaf space $Z$ is a compact complex orbifold $\mathcal{Z}$ with a Kähler metric $h$ and Kähler form $\omega$ which defines a class $[\omega]$ in $H_{\text {orb }}^{2}(\mathcal{Z}, \mathbb{Z})$ in such a way that $\pi:(M, g) \longrightarrow(\mathcal{Z}, h)$ is an orbifold Riemannian submersion. The fibers of $\pi$ are totally geodesic submanifolds of $\mathcal{S}$ diffeomorphic to $S^{1}$.

(ii) The underlying complex space $Z$ is a polarized normal projective algebraic variety with at worst quotient singularities.

(iii) The orbifold $\mathcal{Z}$ is Fano if and only if $\operatorname{Ric}_{g}>-2$. In this case $Z$ is simply connected, and as an algebraic variety is uniruled with Kodaira dimension $\kappa(Z)=-\infty$.

(iv) $(M, g)$ is Sasakian-Einstein iff $(\mathcal{Z}, h)$ is Kähler-Einstein with scalar curvature $4 n(n+1)$.

Here $H_{\text {orb }}^{*}(\mathcal{Z}, \mathbb{Z})$ is the orbifold cohomology due to Haefliger Hae84 (cf. BG00] for more detail), which rationally, but not integrally, coincides with the ordinary cohomology $H^{*}(Z, \mathbb{Q})$. The important point is that $H_{\text {orb }}^{2}(\mathcal{Z}, \mathbb{Z})$ classifies circle V-bundles (orbibundles) over the orbifold $\mathcal{Z}$. We also have an Inversion Theorem which allows one to construct a Sasakian manifold (orbifold) from a polarized compact Kähler orbifold.

Theorem 3.4: $\mathrm{BG00}$ Let $(\mathcal{Z}, \omega)$ be a compact Kähler orbifold with $[\omega] \in H_{\text {orb }}^{2}(\mathcal{Z}, \mathbb{Z})$, and let $\pi: M \longrightarrow \mathcal{Z}$ be the $S^{1} V$-bundle over $\mathcal{Z}$ whose orbifold first Chern class is $[\omega]$. Suppose further that the local uniformizing groups of the orbifold inject into $S^{1}$, the group of the bundle, and that $[\omega]$ is a generator in $H_{\text {orb }}^{2}(\mathcal{Z}, \mathbb{Z})$. Then $M$ is a compact simply connected manifold which admits a Sasakian structure $\mathcal{S}$ whose basic first Chern class $c_{1}\left(\mathcal{F}_{\xi}\right)$ equals 
$\pi^{*} c_{1}^{\text {orb }}(\mathcal{Z})$. Furthermore, there is a 1-1 correspondence between compatible Kähler orbifold metrics on $\mathcal{Z}$ in the same Kähler class and homologous (with respect to the basic cohomology) Sasakian structures on $M$.

Here we refer to the the basic cohomology $H_{B}^{*}(\mathcal{F})$ associated with a Riemannian foliation (cf. [Ton97] for details). We now have

3.2. A Brief Review of Orbifolds. Since in the above description the orbifold structure of $\mathcal{Z}$ is crucial, we give a very brief review concentrating on the important distinctions with the geometry of manifolds or varieties. A given algebraic variety may have many inequivalent orbifold structures. In categorical language the topos of sheaves on $\mathcal{Z}$ may be non-standard.

Orbifolds were invented by Satake [Sat56, Sat57] under the name V-manifold, and later rediscovered and renamed by Thurston [Thu79]. Since here we are only concerned with complex orbifolds, we give the definition in this case only.

Definition 3.5: $A$ complex orbifold $\mathcal{Z}$ is a complex space $Z$ together with a covering of charts $\mathcal{U}=\left\{\tilde{U}_{i}\right\}$, called local uniformizing charts, such that the natural projections $\varphi_{i}$ : $\tilde{U}_{i} \longrightarrow U_{i}=\mathbb{C}^{n} / \Gamma_{i}$ cover $Z$, and $\Gamma_{i}$ is a finite subgroup of $U(n)$, called a local uniformizing group that satisfy certain compatibility conditions. (See [Sat57, Bai57] for details).

We often write the orbifold as $\mathcal{Z}=(Z, \mathcal{U})$, or just $\mathcal{Z}$. The local geometry on orbifolds is much the same as on manifolds, keeping in mind that one works equivariantly on the local uniformizing covers. However, working on these local uniformizing covers causes certain shifts in the presence of branch divisors as we shall see below. For complete definitions and details concerning orbibundles (V-bundles), orbisheaves, etc., we refer to the literature Sat57, Bai56, Bai57, MM03. A fairly comprehensive treatment will also appear in our forthcoming book BG05. Suffice it to say at this stage that an orbi-object is a family of geometric objects defined on the local uniformizing charts that satisfy the correct compatibility conditions. For example, the canonical line bundle $K_{\mathcal{Z}}^{o r b}$ of the orbifold $\mathcal{Z}$ is a family of line bundles, one on each chart $\tilde{U}_{i}$, which is the highest exterior power of the holomorphic cotangent bundle. Actually, instead of the canonical orbibundle, we work with canonical divisors which by abuse of notation we denote also by $K_{\mathcal{Z}}^{\text {orb }}$. In order to understand their relation with ordinary canonical divisors we need to consider the orbifold singular set and its affect on divisors.

Definition 3.6: The orbifold singular set $\Sigma(\mathcal{Z})$ is the subset of $Z$ where $\Gamma_{i} \neq i d$.

We shall always assume the orbifold is effective, that is that the local uniformizing groups act effectively. It is easy to see that the orbifold regular set $Z / \Sigma(\mathcal{Z})$ is a dense open subset of $Z$. Generally, the orbifold singular set $\Sigma(\mathcal{Z})$ differs from the usual algebro-geometric singular set. This is due to the well known fact that quotients of a complex space under reflections in hyperplanes are smooth. So the usual singularity set of an algebraic variety is generally only a subset of $\Sigma(\mathcal{Z})$.

Definition 3.7: $A$ branch divisor $\Delta$ of an orbifold $\mathcal{Z}=(Z, \mathcal{U})$ is a $\mathbb{Q}$-divisor on $Z$ of the form

$$
\Delta=\sum_{\alpha}\left(1-\frac{1}{m_{\alpha}}\right) D_{\alpha}
$$


where $D_{\alpha}$ is a Weil divisor on $Z$ that lies in the orbifold singular locus $\Sigma^{\text {orb }}(Z)$, and $m_{\alpha}$ is the gcd of the orders of the local uniformizing groups taken over all points of $D_{\alpha}$, and is called the ramification index of $D_{\alpha}$.

A crucial relation for us is given by

Lemma 3.8: Orbifold canonical divisors $K_{\mathcal{Z}}^{o r b}$ and the usual canonical divisors $K_{Z}$ are related by

$$
K_{\mathcal{Z}}^{\text {orb }} \equiv \varphi^{*} K_{Z}+\sum_{\alpha}\left(1-\frac{1}{m_{\alpha}}\right) \varphi^{*} D_{\alpha}
$$

In particular $K_{\mathcal{Z}}^{o r b} \equiv \varphi^{*} K_{Z}$ if and only if there are no branch divisors.

Here $\equiv$ denotes linear equivalence, and $\varphi=\sqcup \varphi_{i}$. As in the usual case the orbifold first Chern class satisfies $c_{1}^{\text {orb }}(\mathcal{Z})=c_{1}\left(-K_{\mathcal{Z}}^{\text {orb }}\right)$; however, generally it is only a rational class which, of course, can differ from the first Chern class of the algebraic variety $Z$. In particular, Fano as an orbifold is different than Fano as an algebraic variety. It is easy to give examples of non-Fano orbifold structures that lie on an algebraic variety that is Fano. It is Fano orbifold structures that interest us here.

\section{BRIESKORN'S WORK}

Ten years after Milnor's famous construction of exotic differential structures on $S^{7}$ Brieskorn [Bri66] showed how one could construct all homotopy spheres $\Sigma^{2 n-1} \in b P_{2 n}$ explicitly.

- Consider an $n+1$-tuple of positive integers $\mathbf{a}=\left(a_{0}, \ldots, a_{n}\right)$ and Brieskorn-Pham polynomials in $\mathbb{C}^{n+1}$ with $a_{i}>1$ :

$$
f=z_{0}^{a_{0}}+\cdots+z_{n}^{a_{n}} .
$$

- Construct the link: $L(\mathbf{a})=f^{-1}(0) \cap S^{2 n+1}$. By the Milnor Fibration Theorem $L(\mathbf{a})$ is $(n-2)$-connected.

4.1. Brieskorn Graph Theorem. To a one associates a graph $G(\mathbf{a})$ whose $n+1$ vertices are labeled by $a_{0}, \ldots, a_{n}$. Two vertices $a_{i}$ and $a_{j}$ are connected if and only if $\operatorname{gcd}\left(a_{i}, a_{j}\right)>1$. Let $G(\mathbf{a})_{e v}$ denote the connected component of $G(\mathbf{a})$ determined by the even integers.

Theorem 4.1: Bri66 The link $L(\mathbf{a})$ is homeomorphic to the $(2 n-1)$ - sphere if and only if either of the following hold:

(i) (i) $G(\mathbf{a})$ contains at least two isolated points, or

(ii) (ii) $G(\mathbf{a})$ contains a unique odd isolated point and $G(\mathbf{a})_{e v}$ has an odd number of vertices with $\operatorname{gcd}\left(a_{i}, a_{j}\right)=2$ for any distinct $a_{i}, a_{j} \in G(\mathbf{a})_{e v}$.

One considers the cases $n=2 m$ and $n=2 m+1$ separately. In particular, consider the polynomials:

$$
f=z_{0}^{6 k-1}+z_{1}^{3}+z_{2}^{2}+\cdots+z_{2 m}^{2}
$$

and

$$
f=z_{0}^{p}+z_{1}^{2}+z_{2}^{2}+\cdots+z_{2 m+1}^{2} \quad(p \text { odd })
$$

These are examples of types (i) and (ii), respectively, of the Graph Theorem. Brieskorn shows that with these two polynomials one can describe all exotic spheres that bound parallelizable manifolds! Using these polynomials we proved BGN03b that all such exotic spheres admit 
Sasakian metrics of positive Ricci curvature, but the existence of Einstein metrics is another matter.

4.2. The Diffeomorphism types. Modulo the problems with $b P_{4 m+2}$ mentioned earlier, Brieskorn determined the diffeomorphism type of any Brieskorn manifold satisfying the conditions of his graph theorem. Assume the conditions of the graph theorem are satisfied, so the link is a homotopy sphere $\Sigma^{2 n+1}$.

- For $b P_{4 m}$ the diffeomorphism type is determined by the Hirzebruch signature $\tau\left(V_{4 m}\right)$ of the Milnor fiber $V_{4 m}$ whose boundary is $\Sigma^{4 m-1}$. Then

$$
\begin{aligned}
\tau\left(V_{4 m}(\mathbf{a})\right) & =\#\left\{\mathbf{x} \in \mathbb{Z}^{2 m+1} \mid 0<x_{i}<a_{i} \text { and } 0<\sum_{j=0}^{2 m} \frac{x_{i}}{a_{i}}<1 \bmod 2\right\} \\
& -\#\left\{\mathbf{x} \in \mathbb{Z}^{2 m+1} \mid 0<x_{i}<a_{i} \text { and } 1<\sum_{j=0}^{2 m} \frac{x_{i}}{a_{i}}<2 \bmod 2\right\}
\end{aligned}
$$

The homotopy sphere $\Sigma_{i}^{4 m-1}$ is then determined by

$$
i=\frac{1}{8} \tau\left(V_{4 m}\left(\Sigma_{i}\right)\right) \bmod \left|b P_{4 m}\right| \text {. }
$$

- For $b P_{4 m+2} \neq 0$ the diffeomorphism type is determined by the so-called Arf invariant:

$$
C\left(V_{4 m+2}(\mathbf{a})\right) \in\{0,1\} .
$$

Then $\Sigma^{4 m+1}$ is the standard sphere if $C\left(V_{4 m+2}(\mathbf{a})\right)=0$, and the Kervaire sphere if $C\left(V_{4 m+2}(\mathbf{a})\right)=$ 1. Furthermore, we obtain the Kervaire sphere if and only if condition (ii) of the Brieskorn Graph Theorem holds and the one isolated point, say $a_{0}$, satisfies $a_{0} \equiv \pm 3 \bmod 8$.

\section{SASAKian GeOmetry On LinKs}

In 1978 Takahashi Tak78 showed that the Brieskorn manifolds $L(\mathbf{a})$ naturally admit Sasakian structures. Recall that a Riemannian manifold $(M, g)$ is Sasakian if its metric cone $\left(M \times \mathbb{R}^{+}, d r^{2}+r^{2} g\right)$ is Kähler.

5.1. The weighted Sasakian structure on links in $S^{2 n+1}$. Let $\mathbf{w}=\left(w_{0}, \cdots, w_{n}\right)$ be a vector whose components are positive integers. The "weighted" Sasakian structure $\left(\xi_{\mathbf{w}}, \eta_{\mathbf{w}}, \Phi_{\mathbf{w}}, g_{\mathbf{w}}\right)$ which in the standard coordinates $\left\{z_{j}=x_{j}+i y_{j}\right\}_{j=0}^{n}$ on $\mathbb{C}^{n+1}=\mathbb{R}^{2 n+2}$ is determined by

$$
\eta_{\mathbf{w}}=\frac{\sum_{i=0}^{n}\left(x_{i} d y_{i}-y_{i} d x_{i}\right)}{\sum_{i=0}^{n} w_{i}\left(x_{i}^{2}+y_{i}^{2}\right)}, \quad \xi_{\mathbf{w}}=\sum_{i=0}^{n} w_{i}\left(x_{i} \partial_{y_{i}}-y_{i} \partial_{x_{i}}\right),
$$

and the standard Sasakian structure $(\xi, \eta, \Phi, g)$ on $S^{2 n+1}$. This gives the weighted Sasakian structure on $S^{2 n+1}$ described previously [BG01].

Now consider a Brieskorn-Pham polynomial

$$
f=z_{0}^{a_{0}}+\cdots+z_{n}^{a_{n}}
$$

such that $a_{i} w_{i}=d$ for each $i$. Then the vector field $\xi_{\mathbf{w}}$ restricts to the link $L(\mathbf{a})$ and the embedding $L(\mathbf{a}) \hookrightarrow S^{2 n+1}$ induces a weighted Sasakian structure on $L(\mathbf{a})$. The integer $d$ is called the weighted degree of $f$. 
The flow of $\xi_{\mathbf{w}}$ gives a locally free $S^{1}$ action on both $L(\mathbf{a})$ and $S^{2 n+1}$. The quotient by this action on $S^{2 n+1}$ is a weighted projective space $\mathbb{P}(\mathbf{w})$, and the quotient on $L(\mathbf{a})$ is a Kähler orbifold $\mathcal{Z}(\mathbf{a})$ and we have a commutative diagram

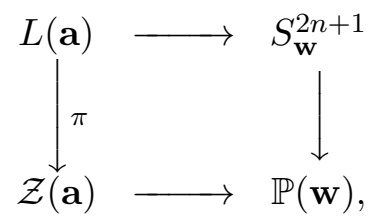

where the horizontal arrows are Sasakian and Kählerian embeddings, respectively, and the vertical arrows are principal $S^{1} \mathrm{~V}$-bundles and orbifold Riemannian submersions.

From the discussion above and Theorem 3.3 we have

Lemma 5.1: Let $L(\mathbf{a})$ be as above. Then the following hold:

(i) The orbifold $\mathcal{Z}(\mathbf{a})$ is Fano (i.e., $c_{1}^{\text {orb }}(\mathcal{Z}(\mathbf{a}))>0$ ) if and only if $|\mathbf{w}|:=\sum_{i} w_{i}>d$.

(ii) $L$ (a) admits a compatible Sasakian-Einstein metric if and only if $\mathcal{Z}(\mathbf{a})$ admits a compatible Kähler-Einstein orbifold metric of scalar curvature $4 n(n-1)$.

Our aim is to prove the existence of Sasakian-Einstein metrics on the links of BrieskornPham singularities which represent homotopy spheres. By Lemma 5.1 this is equivalent to proving the existence of Kähler-Einstein orbifold metrics on the quotient orbifolds. We also want to know that inequivalent Sasakian-Einstein structures imply inequivalent Einstein metrics.

Lemma 5.2: Let $\mathcal{S}=(\xi, \eta, \Phi, g)$ and $\mathcal{S}^{\prime}=\left(\xi^{\prime}, \eta^{\prime}, \Phi^{\prime}, g\right)$ be non-conjugate Sasakian structures on $L(\mathbf{a})$ sharing the same Riemannian metric $g$ which is not of constant curvature. Suppose also that $L(\mathbf{a})$ is a homotopy sphere and satisfies $|\mathbf{w}|-d<\frac{n}{2} \min \left\{w_{i}\right\}$. Then $\mathcal{S}=\mathcal{S}^{\prime}$.

This follows essentially from Lemma 3.2. The inequality implies that the orbifold $\mathcal{Z}(\mathbf{a})$ does not admit a holomorphic contact structure which implies that $\mathcal{S}$ and $\mathcal{S}^{\prime}$ cannot be part of a 3-Sasakian structure [BG97. All of the Einstein metrics obtained in BGK03, BGKT03] satisfy the inequality in Lemma 5.2 .

5.2. Existence: Solving the Monge-Ampère equation. The continuity method developed over the years by a number of mathematicians (Aubin,Yau, Tian-Yau, Tian, Siu, Nadel, Demailly-Kollár) proves the existence of a Kähler-Einstein metric on a compact orbifold by proving an openness-closedness condition. Openness follows from an inverse function theorem argument and, in general, there are obstructions to the closedness. Our aim is to find a family of functions $\phi_{t}$ and numbers $C_{t}$ for $t \in[0,1]$, normalized by the condition $\int_{\mathcal{Z}} \phi_{t} \omega_{0}^{n-1}=0$, such that they satisfy the Monge-Ampère equation

$$
\log \frac{\left(\omega_{0}+\frac{i}{2 \pi} \partial \bar{\partial} \phi_{t}\right)^{n-1}}{\omega_{0}^{n-1}}+t\left(\phi_{t}+f\right)+C_{t}=0 .
$$

We start with $\phi_{0}=0, C_{0}=0$ and if we can reach $t=1$, we get a Kähler-Einstein metric

$$
\omega_{1}=\omega_{0}+\frac{i}{2 \pi} \partial \bar{\partial} \phi_{1} .
$$

To find such a Kähler-Einstein metric we require that the values of $t$ for which the MongeAmpère equation is solvable approach a critical value $t_{0} \in[0,1]$, a subsequence of the $\phi_{t}$ 
converges to a function $\phi_{t_{0}}$ which is the sum of a $C^{\infty}$ and of a plurisubharmonic function. As discussed by Tian [Tia87] we only need to prove that

$$
\int_{\mathcal{Z}} e^{-\gamma \phi_{t_{0}}} \omega_{0}^{n-1}<+\infty \text { for some } \gamma>\frac{n-1}{n}
$$

Alternatively this condition can be phrased in terms of multiplier ideal sheaves and a condition known in algebraic geometry as Kawamata log terminal (klt). Using Demailly and Kollár DK01] we get a Kähler-Einstein metric on $\mathcal{Z}(\mathbf{a})$, hence an Sasakian-Einstein metric on $L(\mathbf{a})$, if there is a $\gamma>\frac{n-1}{n}$ such that for every weighted homogeneous polynomial $g \neq 0$ of degree $s(|\mathbf{w}|-d)$, the function $|g|^{-\frac{\gamma}{s}}$ is locally $L^{2}$. We remark that the uniform boundedness also needs to be proven along singular orbifold divisors. In algebraic geometry this is accomplished by the inversion of adjunction.

Putting this together we arrive at our main operational result.

Theorem 5.3: BGK03 The orbifold $\mathcal{Z}(\mathbf{a})$ is Fano and has a Kähler-Einstein metric if it satisfies the condition

$$
1<\sum_{i=0}^{n} \frac{1}{a_{i}}<1+\frac{n}{n-1} \min _{i, j}\left\{\frac{1}{a_{i}}, \frac{1}{b_{i} b_{j}}\right\} .
$$

where $b_{j}=\operatorname{gcd}\left(a_{j}, C^{j}\right)$ with $C^{j}=\operatorname{lcm}\left(a_{i}: i \neq j\right)$.

The left hand inequality is the Fano condition while the right hand inequality is the klt condition.

We can deform Brieskorn-Pham polynomials by adding arbitrary monomials of weighted degree $d$. This gives large moduli spaces of Kähler-Einstein metrics on $\mathcal{Z}(\mathbf{a})$ which is represented projectively by the deformed weighted homogeneous polynomials. Then one obtains moduli spaces of Sasakian-Einstein metrics by using Lemma 3.2. The parameter count comes from writing down all possible monomials satisfying the requisite conditions, and using the automorphisms of the weighted projective spaces $\mathbb{P}(\mathbf{w})$ to obtain normal forms. A large lower bound on the dimension of the Sasakian-Einstein moduli space then arises from a large ged between two or more of the integers in the sequence $\mathbf{a}=\left(a_{0}, \cdots, a_{n}\right)$.

5.3. Satisfying the Inequalities. We are interested in finding sequences of integers $\mathbf{a}=$ $\left(a_{0}, \cdots, a_{n}\right)$ with $n \geqslant 3$ that satisfy the inequalities of Theorem 5.2 as well as the conditions of the Brieskorn Graph Theorem. For low values of $n$ the inequalities of Theorem 5.3 are easily solved on a computer. However, it is not only instructive to obtain some solutions by hand, but we can prove some interesting results by judicious choices of sequences. We consider a very important sequence that does not satisfy the inequalities, but marks the borderline of the left hand inequality. It is the so-called extremal sequence or Sylvester's sequence [GKP89] determined by the recursion relation

$$
c_{k+1}=1+c_{1} \cdots c_{k}=c_{k}^{2}-c_{k}+1
$$

beginning with $c_{1}=2$. It starts as

$$
2,3,7,43,1807,3263443,10650056950807, \ldots
$$

The importance of this sequence is that it satisfies

$$
\sum_{i=0}^{n} \frac{1}{c_{i}}=1-\frac{1}{c_{0} \cdots c_{n}} .
$$


So we see that sequences of the form $\mathbf{a}=\left(a_{0}=c_{0}, \ldots, a_{n-1}=c_{n-1}, a_{n}\right)$ satisfy the left hand inequality as long as $a_{n}<c_{0} \cdots c_{n-1}$. Furthermore, by construction the first $n$ elements of such sequences are all relatively prime to each other, so the conditions of the Brieskorn Graph Theorem are automatically satisfied. It remains to analyze the right hand inequality.

The troublesome part of the inequalities in Theorem 5.3 is the computation of the $b_{i}$. However, for general sequences if the $a_{i}$ are all pairwise relatively prime, $b_{i}=1$ for all $i$, so again with the order $a_{0}<a_{1}<\cdots<a_{n}$ we see that in this case the inequalities become

$$
1<\sum_{i=0}^{n} \frac{1}{a_{i}}<1+\frac{n}{n-1} \frac{1}{a_{n}} .
$$

Applying this to our special sequence $\mathbf{a}=\left(a_{0}=c_{0}, \ldots, a_{n-1}=c_{n-1}, a_{n}\right)$, we see that the right hand inequality automatically holds as long as $a_{n}$ is relatively prime to the $c_{i}$ 's, a condition that is easy to satisfy for all $n$. This gives a huge number of examples. However, since the $a_{i}$ 's are pairwise relatively prime these sequences give no moduli.

Another approach which gives a large number of sequences, but now some will have moduli, is to notice that $b_{i} \leqslant a_{i}$, so it is sufficient to satisfy the following stronger restriction:

$$
1<\sum_{i=0}^{n} \frac{1}{a_{i}}<1+\frac{n}{n-1} \min _{i, j}\left\{\frac{1}{a_{i} a_{j}}\right\}=1+\frac{n}{n-1} \cdot \frac{1}{a_{n-1} a_{n}} .
$$

By direct computation this is satisfied if $c_{n}-c_{n-1}<a_{n}<c_{n}$. At least a third of these numbers are relatively prime to $a_{1}=2$ and to $a_{2}=3$, thus we conclude

Proposition 5.4: BGK03] Our methods yield at least $\frac{1}{3}\left(c_{n}-1\right) \geqslant \frac{1}{3}(1.264)^{2^{n}}-0.5$ inequivalent families of Sasakian-Einstein metrics on (standard and exotic) $(2 n-1)$-spheres.

If $2 n-1 \equiv 1 \bmod 4$ then all these metrics are on the standard sphere. If $2 n-1 \equiv 3$ mod 4 then all these metrics are on on both standard and exotic spheres but we cannot say anything in general about their distribution.

ExAmple 5.5: Consider sequences of the form $\mathbf{a}=(2,3,7, m)$. By explicit calculation, the corresponding link $L(\mathbf{a})$ gives a Sasakian-Einstein metric on $S^{5}$ if $5 \leqslant m \leqslant 41$ and $m \neq 7$ is relatively prime to at least two of $2,3,7$. This is satisfied in 27 cases. For example, the sequence $\mathbf{a}=(2,3,7,35)$ is especially noteworthy. If $C(u, v)$ is any sufficiently general homogeneous septic polynomial, then the link of

$$
x_{1}^{2}+x_{2}^{3}+C\left(x_{3}, x_{4}^{5}\right)
$$

also gives a Sasakian-Einstein metric on $S^{5}$. The relevant automorphism group of $\mathbb{C}^{4}$ is

$$
\left(x_{1}, x_{2}, x_{3}, x_{4}\right) \mapsto\left(x_{1}, x_{2}, \alpha_{3} x_{3}+\beta x_{4}^{5}, \alpha_{4} x_{4}\right) .
$$

Hence we get a $2(8-3)=10$ real dimensional family of Sasakian-Einstein metrics on $S^{5}$.

ExAmple 5.6: A similar analysis to the previous example shows, for example, that the sequence $\mathbf{a}=(2,3,7,43,43 \cdot 31)$ gives a standard 7 -sphere with a $2(44-3)=82$-dimensional family of Sasakian-Einstein metrics on $S^{7}$. Similarly, the sequence $\mathbf{a}=(2,3,7,43,43 \cdot 39)$ gives a $2(44+4-5)=86$ parameter family of Sasakian-Einstein metrics on the exotic sphere $\Sigma_{6}$. In these examples we use a computer to calculate the signature of $V_{8}$ using Brieskorn's combinatorial formula 4.1 .

The results given in Theorems 1.2 and 1.3 are obtained by computer by inputing sequences $\mathbf{a}=\left(a_{0}, \cdots, a_{n}\right)$ and doing searches for those satisfying the conditions of Theorems 4.1 and 5.3. We determined the diffeomorphism type using the Brieskorn formula or a modification 
of it in terms of sums of products of cotangents due to Zagier. By Proposition 5.4 the number of deformation classes grows double exponentially with $n$, and it is easy to see that the number of effective parameters as well grows double exponentially. For example, this way one obtains 68 inequivalent families of Sasakian-Einstein metrics on $S^{5}$ (this computation can actually be easily done without a computer). The largest family has 10 parameters. A partial computer search yielded more than $3 \cdot 10^{6}$ cases for $S^{9}$ and more than $10^{9}$ cases for $S^{13}$, including a 21300113901610 -dimensional family. Previously, the only known Einstein metric on $S^{13}$ was the standard one.

Similar results for certain simply connected rational homology spheres were obtained in BG03a]. However, the double exponential growth is now replaced by just single exponential growth. It should should be mentioned that a non-vanishing second Stiefel-Whitney class $w_{2}$ is an obstruction for a simply connected manifold to admit a Sasakian-Einstein metric BG99. Now Smale Sma62 has classified the simply connected spin 5-manifolds, and the natural question arise whether all simply connected 5-manifolds, or even all simply connected rational homology 5-spheres admit a Sasakian-Einstein metric. Infinite series of rational homology 5 -spheres that do admit a Sasakian-Einstein metric were given in BG03a. However, recently Kollár Kol04a has shown that there is a torsion obstruction to admitting even a Sasakian structure. So not all simply connected, spin, rational homology 5-spheres can admit a Sasakian-Einstein metric.

\section{Open Problems}

- Conjecture: All homotopy spheres which bound parallelizable manifolds admit SasakianEinstein metrics.

- Let $K_{\text {min }}$ denote the minimal value of the sectional curvature. Can one obtain an estimate for $K_{\min }$ ? Or better yet, a formula in terms of the weights and degree of the BP polynomial?

- Is there a bound on the dimension of the moduli space of Sasakian-Einstein metrics on a given manifold, (or more generally for appropriately normalized Einstein metrics) on a given manifold? Find formulae that depend only on dimension. Recall that $S^{13}$ has a moduli space of Sasakian-Einstein metrics of dimension greater than $2.1 \times 10^{13}$.

- How is the moduli space of Sasakian-Einstein metrics related the full moduli space of Einstein metrics?

- Does the moduli space of Sasakian-Einstein metrics have an infinite number of components? This is true for the moduli space of deformation classes of positive Sasakian structures on spheres BGN03b, using work of Morita Mor75, and Ustilovsky Ust99] on distinct contact structures. However, our proofs of Sasakian-Einstein metrics on spheres only yield a finite number of deformation classes.

- Does the dimension of a component depend on $K_{\min }$ ?

\section{REFERENCES}

[Bai56] W. L. Baily, The decomposition theorem for V-manifolds, Amer. J. Math. 78 (1956), 862-888. MR 20 \#6537

[Bai57] _ On the imbedding of $V$-manifolds in projective space, Amer. J. Math. 79 (1957), 403-430. MR $20 \# 6538$ 
[BG97] C. P. Boyer and K. Galicki, The twistor space of a 3-Sasakian manifold, Internat. J. Math. 8 (1997), no. 1, 31-60. MR 98e:53072

[BG99] _ 3-Sasakian manifolds, Surveys in differential geometry: essays on Einstein manifolds, Surv. Differ. Geom., VI, Int. Press, Boston, MA, 1999, pp. 123-184. MR 2001m:53076

[BG00] _ On Sasakian-Einstein geometry, Internat. J. Math. 11 (2000), no. 7, 873-909. MR 2001k:53081

[BG01] _ New Einstein metrics in dimension five, J. Differential Geom. 57 (2001), no. 3, 443-463. MR 2003b: 53047

[BG03a] C. P. Boyer and Galicki, Einstein Metrics on Rational Homology Spheres, arXiv:math.DG/0311355 (2003).

[BG03b] Charles P. Boyer and Krzysztof Galicki, New Einstein metrics on $8 \#\left(S^{2} \times S^{3}\right)$, Differential Geom. Appl. 19 (2003), no. 2, 245-251. MR 2002662

[BG05] C. P. Boyer and K. Galicki, Sasakian Geometry, Oxford Mathematical Monographs, Oxford University Press, to appear, Oxford, 2005.

[BGK03] C. P. Boyer, K. Galicki, and J. Kollár, Einstein Metrics on Spheres, to appear in Ann. of Math., arXiv:math.DG/0309408. (2003).

[BGKT03] C. P. Boyer, K. Galicki, J. Kollár, and E. Thomas, Einstein Metrics on Exotic Spheres in Dimensions 7,11, and 15, to appear in Experimental Mathematics, arXiv:math.DG/0311293 (2003).

[BGM93a] C. P. Boyer, K. Galicki, and B. M. Mann, 3-Sasakian manifolds, Proc. Japan Acad. Ser. A Math. Sci. 69 (1993), no. 8, 335-340. MR 95a:53066

[BGM93b] _ Quaternionic reduction and Einstein manifolds, Comm. Anal. Geom. 1 (1993), no. 2, 229-279. MR 95c:53056

[BGM94] The geometry and topology of 3-Sasakian manifolds, J. Reine Angew. Math. 455 (1994), 183-220. MR 96e:53057

[BGMR98] C. P. Boyer, K. Galicki, B. M. Mann, and E. G. Rees, Compact 3-Sasakian 7-manifolds with arbitrary second Betti number, Invent. Math. 131 (1998), no. 2, 321-344. MR 99b:53066

[BGN02] C. P. Boyer, K. Galicki, and M. Nakamaye, Sasakian-Einstein structures on $9 \#\left(S^{2} \times S^{3}\right)$, Trans. Amer. Math. Soc. 354 (2002), no. 8, 2983-2996 (electronic). MR 2003g:53061

[BGN03a] _ On the geometry of Sasakian-Einstein 5-manifolds, Math. Ann. 325 (2003), no. 3, 485524. MR 2004b:53061

[BGN03b] _ Sasakian geometry, homotopy spheres and positive Ricci curvature, Topology 42 (2003), no. 5, 981-1002. MR 1978045

[BJM84] M. G. Barratt, J. D. S. Jones, and M. E. Mahowald, Relations amongst Toda brackets and the Kervaire invariant in dimension 62, J. London Math. Soc. (2) 30 (1984), no. 3, 533-550. MR MR810962 (87g:55025)

[BK78] J-P. Bourguignon and H. Karcher, Curvature operators: pinching estimates and geometric examples, Ann. Sci. École Norm. Sup. (4) 11 (1978), no. 1, 71-92. MR 58 \#12829

[Bla76] D. E. Blair, Contact manifolds in Riemannian geometry, Springer-Verlag, Berlin, 1976, Lecture Notes in Mathematics, Vol. 509. MR 57 \#7444

[Bla02] _ Riemannian geometry of contact and symplectic manifolds, Progress in Mathematics, vol. 203, Birkhäuser Boston Inc., Boston, MA, 2002. MR 2002m:53120

[Böh98] Ch. Böhm, Inhomogeneous Einstein metrics on low-dimensional spheres and other lowdimensional spaces, Invent. Math. 134 (1998), no. 1, 145-176. MR 99i:53046

[Bri66] E. Brieskorn, Beispiele zur Differentialtopologie von Singularitäten, Invent. Math. 2 (1966), 1-14. MR 34 \#6788

[Bro69] William Browder, The Kervaire invariant of framed manifolds and its generalization, Ann. of Math. (2) 90 (1969), 157-186. MR MR0251736 (40 \#4963)

[BW58] W. M. Boothby and H. C. Wang, On contact manifolds, Ann. of Math. (2) 68 (1958), 721-734. MR 22 \#3015

[DK01] J-P. Demailly and J. Kollár, Semi-continuity of complex singularity exponents and KählerEinstein metrics on Fano orbifolds, Ann. Sci. École Norm. Sup. (4) 34 (2001), no. 4, 525-556. MR 2002e:32032

[GKP89] Ronald L. Graham, Donald E. Knuth, and Oren Patashnik, Concrete mathematics, AddisonWesley Publishing Company Advanced Book Program, Reading, MA, 1989, A foundation for computer science. MR 91f:00001 
[Hae84] A. Haefliger, Groupoïdes d'holonomie et classifiants, Astérisque (1984), no. 116, 70-97, Transversal structure of foliations (Toulouse, 1982). MR 86c:57026a

[Hat63] Y. Hatakeyama, Some notes on differentiable manifolds with almost contact structures, Osaka Math. J. (2) 15 (1963), 176-181. MR 27 \#705

[Jen73] G. R. Jensen, Einstein metrics on principal fibre bundles, J. Differential Geometry 8 (1973), 599-614. MR 50 \#5694

[JK01] J. M. Johnson and J. Kollár, Kähler-Einstein metrics on log del Pezzo surfaces in weighted projective 3-spaces, Ann. Inst. Fourier (Grenoble) 51 (2001), no. 1, 69-79. MR 2002b:32041

[KM63] M. A. Kervaire and J. W. Milnor, Groups of homotopy spheres. I, Ann. of Math. (2) 77 (1963), 504-537. MR 26 \#5584

[Kob63] S. Kobayashi, Topology of positively pinched Kaehler manifolds, Tôhoku Math. J. (2) 15 (1963), 121-139. MR 27 \#4185

[Kol04a] J. Kollár, Einstein metrics on 5-dimensional Seifert bundles, arXiv:math.DG/0408184 (2004).

[Kol04b] _ Einstein metrics on connected sums of $S^{2} \times S^{3}$, arXiv:math.DG/0402141 (2004).

[Lan00] T. Lance, Differentiable structures on manifolds, Surveys on surgery theory, Vol. 1, Ann. of Math. Stud., vol. 145, Princeton Univ. Press, Princeton, NJ, 2000, pp. 73-104. MR 2001d:57035

[Mil56] J. Milnor, On manifolds homeomorphic to the 7-sphere, Ann. of Math. (2) 64 (1956), 399-405. MR 18,498d

[MM03] I. Moerdijk and J. Mrčun, Introduction to foliations and Lie groupoids, Cambridge Studies in Advanced Mathematics, vol. 91, Cambridge University Press, Cambridge, 2003. MR 2012261

[Mor75] Shigeyuki Morita, A topological classification of complex structures on $S^{1} \times S^{2 n-1}$, Topology 14 (1975), 13-22. MR MR0405444 (53 \#9237)

[MT67] Mark Mahowald and Martin Tangora, Some differentials in the Adams spectral sequence, Topology 6 (1967), 349-369. MR MR0214072 (35 \#4924)

[Sas60] S. Sasaki, On differentiable manifolds with certain structures which are closely related to almost contact structure. I, Tôhoku Math. J. (2) 12 (1960), 459-476. MR 23 \#A591

[Sat56] I. Satake, On a generalization of the notion of manifold, Proc. Nat. Acad. Sci. U.S.A. 42 (1956), 359-363. MR 18,144a

[Sat57] _ The Gauss-Bonnet theorem for V-manifolds, J. Math. Soc. Japan 9 (1957), 464-492. MR 20 \#2022

[Sma62] S. Smale, On the structure of 5-manifolds, Ann. of Math. (2) 75 (1962), 38-46. MR 25 \#4544

[Tak78] T. Takahashi, Deformations of Sasakian structures and its application to the Brieskorn manifolds, Tôhoku Math. J. (2) 30 (1978), no. 1, 37-43. MR 81e:53024

[Tan70] S. Tanno, On the isometry groups of Sasakian manifolds, J. Math. Soc. Japan 22 (1970), 579-590. MR 42 \#6755

[Thu79] W. Thurston, The geometry and topology of 3-manifolds, Mimeographed Notes, Princeton University, 1979.

[Tia87] G. Tian, On Kähler-Einstein metrics on certain Kähler manifolds with $C_{1}(M)>0$, Invent. Math. 89 (1987), no. 2, 225-246. MR 88e:53069

[Ton97] P. Tondeur, Geometry of foliations, Monographs in Mathematics, vol. 90, Birkhäuser Verlag, Basel, 1997. MR 98d:53037

[Ust99] Ilya Ustilovsky, Infinitely many contact structures on $S^{4 m+1}$, Internat. Math. Res. Notices (1999), no. 14, 781-791. MR MR1704176 (2000f:57028)

[Wan99] McKenzie Y. Wang, Einstein metrics from symmetry and bundle constructions, Surveys in differential geometry: essays on Einstein manifolds, Surv. Differ. Geom., VI, Int. Press, Boston, MA, 1999, pp. 287-325. MR MR1798614 (2002b:53070)

[YK84] K. Yano and M. Kon, Structures on manifolds, Series in Pure Mathematics, vol. 3, World Scientific Publishing Co., Singapore, 1984. MR 86g:53001

[Zil82] W. Ziller, Homogeneous Einstein metrics on spheres and projective spaces, Math. Ann. 259 (1982), no. 3, 351-358. MR 84h:53062

Department of Mathematics and Statistics, University of New Mexico, Albuquerque, NM 87131.

E-mail address: cboyer@math.unm.edu

E-mail address: galicki@math.unm.edu 